\title{
Antibiotic resistance associated with air quality and transferred by airborne particulate matter
}

\section{Zhen-Chao Zhou}

Zhejiang University

Yang Liu

Zhejiang University

Ze-Jun Lin

Zhejiang University

Xin-Yi Shuai

Zhejiang University

Lin Zhu

Zhejiang University

\section{Lan Xu}

Zhejiang University

Ling-Xuan Meng

Zhejiang University

\section{Yu-Jie Sun}

Zhejiang University

Hong Chen ( $\square$ chen_hong@zju.edu.cn )

Zhejiang University https://orcid.org/0000-0003-3226-4054

\section{Research}

Keywords: air quality, antibiotic resistance, particulate matter, transfer

Posted Date: February 12th, 2020

DOI: https://doi.org/10.21203/rs.2.23315/v1

License: (a) (i) This work is licensed under a Creative Commons Attribution 4.0 International License.

Read Full License 


\section{Abstract}

Background: Environmental spread of antibiotic resistance has become a public health problem, but the relationship between air quality and antibiotic resistance has not been fully investigated, the effect and mechanism of air pollutants on horizontal genes transfer of antibiotic resistance are rarely mentioned.

Results: In the present study, significant correlation $(P<0.05)$ between resistance rates of 10 clinical antibiotic-resistant pathogens and number of days when air quality index was below 100 were observed in an extensive survey from 2014 to 2017 in China. In addition, characterization of antibiotic resistance genes (ARGs), mobile genetic elements (MGEs), and bacterial communities in airborne particulate matter (PM), dust, and human airway sputum samples were profiled in a hospital. PM 2.5 and PM 10 were shown to contain ARGs and MGEs high relative abundance and diversity index. Importantly, transferable multi-resistant plasmids were identified from air, e.g., pTAir-3 with 26 MGEs and 10 ARGs, using conjugative mating assays and nanopore sequencing. Furthermore, PM 2.5 and PM 10 significantly enhanced the conjugative transfer frequencies between bacteria, via inducing increased levels of reactive oxygen species and cell membrane permeability; upregulated gene expression levels were confirmed by genome-wide RNA sequencing.

Conclusions: These findings provide a new perspective on antibiotic resistance research and have profound implications for antibiotic resistance control in clinics and environments.

\section{Introduction}

Antibiotic resistance is a serious threat to public health [1]. Diverse and abundant antibiotic resistance genes (ARGs) are widespread in most environments (e.g., air, water, soil, remote and pristine glacier and caves, urban sewage, and drinking water) [2-11]. Horizontal gene transfer (HGT) is a major driver for disseminating ARGs within and across bacterial genera, via mobile genetic elements (MGEs), such as plasmids, integrons, transposes, and prophages [12]. In this regard, conjugation is the most dominant HGT mechanism to transfer DNA between donor and recipient bacteria [13]. Increasing antibiotic-resistant bacteria (ARB) infections and multi-environmental spreading of ARGs are associated with increasing patient hospitalization and mortality rates [14-17].

Antibiotic resistance and air pollution are included in the WHO reports "ten threats to global health in $2019 "$ ", but $90 \%$ of people breathe polluted air every day [1]. Microscopic pollutants infiltrate respiratory and circulatory systems, whose antibiotic resistance potential might exacerbate morbidity and mortality. ARGs are found in a number of aerial environments including hospitals, cattle feed yards, sewage treatment plants, and urban and rural areas [18-25]. These researches demonstrated ARGs presence and abundance in air environments, however, little is known about the linkage between air quality and antibiotic resistance, and the effects and mechanisms of air pollutants on ARGs spread. To address these issues, this study tested the hypothesis that air quality correlates with antibiotic resistance, and particulate matter (PM) promotes HGT of ARGs. Antibiotic resistance and air quality data across different 
geographical regions in China from 2014 to 2017 were evaluated, including correlation between the number of days with good air quality (here defined as number of days when air quality index is below 100) and antibiotic resistance rates (here defined as percent of pathogens resistant to a particular antibiotic). Furthermore, Sampling sets were designed in a hospital to investigate ARGs, MGEs, and bacteria in the air, dust, and human airway sputum samples, as well as conjugation mating system to identify the effects of $\mathrm{PM}_{2.5}$ and $\mathrm{PM}_{10}$ on ARGs conjugation transfer and mechanisms. The findings provided a comprehensive view of ARGs and MGEs in a hospital aerial environment, and shed light on the relationship between air pollutions and antibiotic resistance.

\section{Materials And Methods}

Data sources and Sampling.

Data sets on air quality and antibiotic resistance (percent resistant) in 13 common pathogens i.e., carbapenem resistant Escherichia coli (CREC), Pseudomonas aeruginosa (CRPA), Acinetobacter baumannii (CRAB), and Klebsiella pneumoniae (CRKP), as well as erythromycin resistant Streptococcus pneumoniae (ERSP), methicillin resistant Staphylococcus aureus (MRSA), and coagulase negative staphylococci (MRCNS), penicillin resistant Streptococcus pneumoniae (PRSP), quinolones resistant E. coli (QREC), third-generation cephalosporin resistant E. coli (3GCREC) and Klebsiella pneumoniae (3GCRKP), and vancomycin resistant Enterococcus faecalis (VREFS) and Enterococcus faecium (VREFM) were downloaded from publicly available data from the National Bureau of Statistics(http://www.stats.gov.cn/) and Antimicrobial Resistance Surveillance System of China(http://www.carss.cn/), respectively. The final data sets of antibiotic resistance bacteria represented over 10 million clinically-relevant bacterial pathogens from over 1.3 thousand hospitals across thirty-two provincial administrative regions of China from 2014 to 2017. The used clinically-relevant bacterial pathogens were mainly derived from sputum specimens (averagely accounting for $41.6 \%$ ), urine specimens (averagely accounting for $17.6 \%$ ) and blood specimens (averagely accounting for $9.5 \%$ ). In China, air quality index (AQI) is divided into six levels, ranging from first-level excellent $(0-50)$, secondlevel good (51-100), third-level mild pollution (101-150), fourth-level moderate pollution (151-200), to fifth-level severe pollution (201-300), and sixth-level severe pollution (more than 300). The number of days with the AQI below 100 can more effectively represent the air quality of a year than the average concentration of pollutants. To characterize ARGs, MGEs, and bacteria community distribution in clinical aerial environment, 70 samples were collected from a hospital during July 27 to August 2 in 2018, including 17 patient airway $(P)$ sputum samples (the samples were collected from volunteers in Inpatient Respiratory Department) and 11 healthy human airway $(\mathrm{H})$ sputum samples, and respiratory-related environmental samples such as 7 indoor dust (ID), 7 outdoor dust (OD), 7 indoor $\mathrm{PM}_{2.5}\left(\mathrm{I}-\mathrm{PM}_{2.5}\right), 7$ outdoor $\mathrm{PM}_{2.5}\left(\mathrm{O}-\mathrm{PM}_{2.5}\right), 7$ indoor $\mathrm{PM}_{10}\left(\mathrm{I}-\mathrm{PM}_{10}\right)$, and 7 outdoor $\mathrm{PM}_{10}\left(\mathrm{O}-\mathrm{PM}_{10}\right)$ samples (detailed sampling protocols, ethics approval, and DNA extraction procedures are described in Supplementary Information Text S1).

High-throughput qPCR. 
ARGs and MGEs were analyzed by high-throughput qPCR (HT-qPCR) using the Takara SmartChip ${ }^{\mathrm{TM}}$. For this, 285 antibiotic resistance gene primer sets for all major classes of antibiotics, 9 transposase gene primer sets, one clinical class 1 integron-integrase gene primer set, and one 16S rRNA gene primer set were evaluated (Table S1) $[6,26]$. The antibiotics resistance genes to aminoglycoside, beta-lactams, fluoroquinolone/quinolone/florfenicol/chloramphenicol/amphenicol (FCA),

macrolide/lincosamide/streptogramin B (MLSB), sulfonamide, and tetracycline, vancomycin, and genes coding multidrug efflux pumps or antibiotic deactivation protein resistance to other antibiotic and bactericide (multidrug) resistance genes were targeted by the 285 ARGs primer sets $[5,27]$ (detailed HTqPCR procedures are described in Supplementary Information Text S2).

Bacterial 16S rRNA gene sequencing.

Bacterial community structures were determined by 16 S rRNA gene sequencing on a HiSeq PE250 platform (Illumina Inc., San Diego, CA). V4 to V5 regions of bacterial 16S rRNA were amplified with the universal primer set 515F (5'- GTGCCAGCMGCCGCGG-3') and 907R (5'-CCGTCAATTCMTTTRAGTTT-3') [28] labeled with unique barcodes (7-nucleotide barcodes) for each sample. All sequences of each sample were screened by filtering adaptor sequences and removing low-quality reads, ambiguous nucleotides, and barcodes. High-quality sequences were analyzed using Quantitative Insights into Microbial Ecology (QIIME) and clustered into operational taxonomic units (OTUs) at $97 \%$ similarity level, using UCLUST.

Physiochemical characterization of $\mathrm{PM}_{2.5}$ and $\mathrm{PM}_{10}$ samples.

$\mathrm{PM}_{2.5}$ and $\mathrm{PM}_{10}$ sample size distributions were evaluated in Zetasizer Nano ZS90 and Mastersizer 2000 equipment (Malvern, UK). Metal elements were analyzed by Inductively coupled plasma emission spectrometer (Perkin Elmer, Norwalk, CT), after acid digestion.

Isolation of airborne conjugative plasmids and nanopore sequencing.

Filter mating assays were applied to capture airborne conjugative plasmids using Escherichia coli NK5449 (nalidixic acid and rifampicin-resistant) as recipients and airborne sample isolations as donors. Airborne sample isolations cultured from collected air sample filters using LB media. Donor and recipient strains initial bacterial concentrations were approximately $10^{8} \mathrm{CFU} / \mathrm{mL}$, which were monitored at an optical density (OD) at $600 \mathrm{~nm}\left(\mathrm{OD}_{600}\right.$ value was approximately 0.8$)$. After culturing for $12 \mathrm{~h}$ at $\mathrm{pH} 7.0$ and $37^{\circ} \mathrm{C}$, suspension was plated on LB agar supplemented with $50 \mathrm{mg} / \mathrm{L}$ kanamycin, $50 \mathrm{mg} / \mathrm{L}$ rifampicin, $100 \mathrm{mg} / \mathrm{L}$ cycloheximide, $100 \mathrm{mg} / \mathrm{L}$ ampicillin, $4 \mathrm{mg} / \mathrm{L}$ ciprofloxacin, $20 \mathrm{mg} / \mathrm{L}$ chloramphenicol, $50 \mathrm{mg} / \mathrm{L}$ gentamicin, $30 \mathrm{mg} / \mathrm{L}$ streptomycin, $10 \mathrm{mg} / \mathrm{L}$ tetracycline, and $50 \mathrm{mg} / \mathrm{L}$ erythromycin, respectively. Transconjugants showed more resistance than recipients, grew on selective LB agar plates, and were identified using denaturing gradient gel electrophoresis (DGGE), following transconjugant plasmids extraction by the Qiagen Midi kit (Qiagen, Germany). Harvested plasmids DNA were detected by agarose gel electrophoresis and quantified by Qubit $\circledast 2.0$ Fluorometer (Thermo Fisher Scientific, Waltham, MA). The whole plasmid genome was sequenced using nanopore sequencing on 
PromethION platform and Illumina NovaSeq PE150 equipments at Beijing Novogene Bioinformatics Technology Co., Ltd.; NCBI reference sequence database, comprehensive antibiotic resistance database, and antibiotic resistance genes database were used to identify antibiotic, biocide, and metal resistance genes. Presence of genomic islands within plasmids was predicted using Island-Viewer 4 and plasmid profiles were created using the SnapGene software 3.2.1.

Conjugation experiments after $\mathrm{PM}_{2.5}$ and $\mathrm{PM}_{10}$ exposure.

An optimized conjugation model to evaluate the conjugative transfer of ARGs between E. coli HB101 and E. coli RP4 was used, as previously reported [29]. E. coli HB101, which harbors RP4 plasmid carrying resistance to tetracycline, ampicillin, and kanamycin, was selected as the donor strain in this study; E. coli NK5449 represented the plasmid recipient. Donor and recipient strains were mixed and treated with $\mathrm{PM}_{2.5} / \mathrm{PM}_{10}$ at $31.25,62.5,125,250$, and $500 \mu \mathrm{g} / \mathrm{mL}$ for $12 \mathrm{~h}$ at $\mathrm{pH} 7.0$ and $37^{\circ} \mathrm{C}[30] . \mathrm{PM}_{2.5}$ and $\mathrm{PM}_{10}$ solutions were prepared as previously reported [30]. Amount of recipient bacteria and transconjugants were counted on LB-selective solid medium as colony-forming units per milliliter (CFU/mL). Transconjugants were identified using DGGE and amplified by PCR with RP4F 5'AAAGCGGACAGCATCAGTAACGAA-3') and RP4R (5'- GAGCTTGGTGGCCGCATAGTGTAG - 3') primers. The conjugative transfer frequency was calculated as the amount of transconjugant cells to recipient cells ratio.

ROS levels evaluation.

Intracellular ROS levels were determined using the 2',7'- dichlorofluorescein diacetate (DCFH-DA) probe (Invitrogen, Carlsbad, CA), according to manufacturer's instructions. A CytoFLEX flow cytometer (Beckman Coulter, Brea, CA) was used to detect ROS at $488 \mathrm{~nm}$ excitation and $525 \mathrm{~nm}$ emission wavelengths, and evaluated by the fluorescence intensity of treated sample and control group ratio. All assays were performed in triplicate.

Transmission electron microscopy (TEM) detection.

Cell membrane permeability was visualized using a JEM-1010 transmission electron microscope (JEOL, Tokyo, Japan) at $75 \mathrm{kV}$ with a CCD camera (detailed procedures are described in Supplementary Information Text S3).

RNA extraction, genome-wide RNA sequencing, and bioinformatics.

Conjugation mating systems were established as described above, using $\mathrm{PM}_{2.5}$ and $\mathrm{PM}_{10}$ at 0.0 (control), 31.25 (low-dosage), 125 (medium-dosage), and $500 \mu \mathrm{g} / \mathrm{mL}$ (high-dosage). After a mating period, QIAGEN miRNeasy Mini Kit (QIAGEN, Germany) was used to extract the total RNA from the mixture, following manufacturer's instructions. RNA samples were analyzed in a Qubit 2.0 (Thermo Fisher Scientific) and quality controlled on an Agilent 2100 bioanalyzer (Agilent Technologies, Palo Alto, CA). cDNA libraries were constructed using the rRNA-depleted RNA by NEBNext® Ultra RNA Library Prep Kit (New England 
Biolabs, Inc., Ipswich, MA). According to supplier's instructions. Sequencing was performed on Illumina NovaSeq PE150 (Illumina Inc.). Gene expression was calculated as fragments per kilobase of a gene per million mapped reads (FPKM). Differences in fold changes between different groups were calculated by log2 fold-change (LFC) between control and airborne PM-treated samples.

\section{Statistical analysis.}

Diversity index, Mantel test, and Procrustes analysis were generated by $\mathrm{R}$ with 'vegan' packages. Heatmaps of China were generated by R with 'mapdata', 'maptools', 'plyr', 'mapproji', 'sp', 'maps', and 'ggplot2' packages. Linear regression analysis graphs were generated by R with 'ggplot2' package. Heatmap was generated by R with 'pheatmap' packages. PCoA (Bray-Curtis distance based) was generated by CANOCO 5. Box and bar charts were generated by OriginPro 9.0 (OriginLab Corp., Northampton, MA). Network analysis was conducted in the Gephi platform and only statistically robust correlations of Spearman's correlation coefficient $(\rho)>0.9$ and significance level $P<0.01$ were used to form the final networks by Frucherman Reingold algorithms [26, 31, 32]. Phylogenetic tree was extracted from the taxa in MEGA7 program, using the maximum-likelihood tree with 500 bootstraps. SourceTracker 1.0.1 was used to estimate the relative contribution of the microbial taxa and ARGs from the source environments to the sink environments by Bayesian methods [33] (details are described in Supplementary Information Text S4).

\section{Results}

\section{Relationship between antibiotic resistance and air quality}

Four-year average number of days when air quality index was below 100 and antibiotic resistance patterns across China from 2014 to 2017 heatmaps were generated (Fig. 1 and S1). Resistance rates of 10 antibiotic resistance pathogens (QREC, CREC, 3GCREC, CRAB, CRPA, MRSA, MRCNS, VREFM, CESP, and 3 GCRKP) showed significant correlation with the number of days when air quality index was below 100 (Fig. 1b, $P<0.05$ ), indicating, less antibiotic resistance pathogens emergence, as the number of clean air days increases. Most antibiotic resistance bacteria used in this correlation are from respiratory samples, so there is rationale for the increase of antibiotic resistance with increased air pollution. Although high air pollution increases respiratory infections, the increase in the antibiotic resistance (percentage of antibiotic resistance bacteria) is unexpected.

\section{Abundant aerial antibiotic resistance genes}

Seventy samples including air, dust, and human airway sputum samples were collected for seven consecutive days to further identify ARGs in air or in its relevant matters, observing differential number and Shannon index of detected ARGs in samples (Fig. 2a and b). Principal coordinate analysis (PCoA) was performed based on ARGs profiles abundance, using the Bray-Curtis dissimilarity index (Fig. 2C). PCoA showed that I-PM $2.5, I-\mathrm{PM}_{10}, 0-\mathrm{PM}_{2.5}, \mathrm{O}-\mathrm{PM}_{10}$, and OD samples formed one cluster on the projection 
plot (Fig. 2C), whereas airway sputum and ID samples formed two separate clusters. I-PM 10 and ID samples contained the highest ARGs abundance with an average of 5.4 copies and the least ARGs abundance with an average of 0.5 copies per $16 \mathrm{~S}$ rDNA gene copies, respectively (Fig. 2d). ARGs abundance had no significant difference between indoor and outdoor samples, whereas ARGs abundances in $\mathrm{PM}_{10}$ and $\mathrm{PM}_{2.5}$ were higher than those in dust and airway sputum samples $(\mathrm{P}<0.05)$. ARGs distribution among 8 different samples is shown in Fig. 2e, whereas that of ARG subtypes are shown in the heatmap (Fig. S2). Genes providing resistance to aminoglycoside and multidrugs were mostly present in dust and air samples. Tetracyclines and MLSB resistance genes contributed to $22 \%$ and $53 \%$ in human airway sputum, respectively.

\section{Mobile genetic elements}

The total abundance of transposase and integron-integrase genes in samples is shown in Figures S3 and S4. $\mathrm{PM}_{10}$ samples contained higher transposase and integron-integrase abundance than others, with an average of 0.4 and 2.9 copies per $16 \mathrm{~S}$ rDNA gene, respectively. Furthermore, ARGs abundance significantly $(P<0.01)$ correlated with that of transposases and integron-integrase genes (Fig. S5).

\section{Microbial Taxa in air}

From the $16 \mathrm{~S}$ ribosomal gene amplicon sequencing, raw data consisted of 3237776 reads for all samples, and quality-filtered data consisted of 2767855 reads with an average value of 43247 reads per sample, corresponding to 829 operational taxonomic units, after filtering and quality control. ID samples harbored more observed species and showed higher bacterial diversity than others (Fig. S6a and b). PCoA results of bacteria communities indicated that airway sputum and other environmental samples formed two separate clusters (Fig. S6c), which was consistent with PCoA of ARGs profiles. In addition, airway sputum samples contained low observed species and bacterial diversity; bacterial communities' composition was significantly different among airway sputum samples and other environmental samples at the phylum level $(P<0.05 ;$ Fig. S6d). Firmicutes were the dominant phylum in the airway sputum samples, accounting for $43 \%$ of total bacterial $16 \mathrm{~S}$ rRNA gene sequences. Proteobacteria was the prevailing phylum in dust and air samples, accounting for $30-65 \%$.

\section{Correlation between ARGs and bacterial community}

Microbial taxa and ARGs-based PCoA plots showed similar sample clustering patterns. To further investigate whether the microbial community correlated with ARG composition, Procrustes analysis and Mantel test were used to correlate both profiles. Results showed that ARG profiles significantly correlated to bacterial compositions and structures (Bray-Curtis, $M^{2}=0.63, r=0.61, P<0.001$, based on 9999 permutations; Fig. S6e). The Mantel test also revealed a significant correlation between ARG profiles and microbial OTUs $(r=0.28, P<0.01)$.

\section{Co-occurrence patterns among ARGs, MGEs, and microbial taxa}


The resultant network consisted of 224 nodes (each node represented a subtype of ARGs, MGEs or microbial taxa) and 440 edges, with a node connectivity of 3.93 (Fig. 3a). The clustering coefficient was 0.73 , and the modularity index was 0.89 (values $>0.4$ ), suggesting that the network had a modular structure $[34,35]$. Based on the modularity class, the entire network was clearly separated into eight major modules. Compared with a random association, clusters of nodes in the same module contained more interactions among themselves than with other nodes. In some modules, some clinical critical microbial taxa were supposed to carry some specific ARGs. For example, Campylobacter spp. positively correlated with ARGs, conferring resistance to fluoroquinolone (vatC-02, mexD), $\beta$-lactams (fox5), tetracycline (tet3601, tetA-01, tetPB-02), and vancomycin (vanSB, vanSE, vanTC-02, vanTE, vanYB) in module I, whereas Staphylococcus spp. positively correlated with ARGs, conferring resistance to $\beta$-lactams (blaZ, mecA), aminoglycoside (aacA_aphD, aadA-1, aadE), and multi-drug (sat4) in module II. The phylogenic tree at the genus level showed the phylogenic relationship of genera and their associated MGEs and ARGs (Fig. 3b).

\section{SourceTracker analysis of bacteria and antibiotic resistance genes}

SourceTracker was used to analyze the relative contribution of environmental bacteria and ARGs to human airway sputum (Fig. S7). SourceTracker analysis has been performed to identify the extent of contribution of each source to the sink, as previously reported [36-38]. It can be used for estimating the relative contributions of taxa and ARGs from exposed environments (sources) in the human airway sputum (sinks). In healthy human airway sputum, I-PM 10 was the highest relative contribution of bacteria from six environments, accounting for $2.5 \%$. In patient airways sputum, O-PM 2.5 and I-PM 2.5 contributed to a higher proportion of bacteria than others, accounting for $3.5 \%$ and $3.6 \%$, respectively. Similar trends were observed when SourceTracker analyses were run on ARG data, whereas the relative contribution of ARGs was significantly $(P<0.05)$ lower than bacterial taxa (Fig. S7b). In the patient airways sputum, O$\mathrm{PM}_{2.5}$ contributed to a higher proportion of ARGs than others, accounting for $1.8 \%$.

\section{Conjugative multi-resistant plasmids in air}

Three conjugative multi-resistant plasmids were demonstrated from air. The plasmid pTAir-3 was 146,145 nucleotides in length, had a GC content of $51 \%$ and carried 26 horizontal transfer genes and 10 antibiotic resistance genes, as well as type IV secretion systems and fimbrial genes (Fig. 4). Nine metal resistance genes (including cusB/F copper, silC/E/R silver, and merA/C/D/E/P cadmium resistance genes) and four antibiotic resistance genes (bleO bleomycin, EamA family, and tetG and tetR tetracycline resistance genes) were present in the genomic island regions spanning nucleotide positions 38656-68101; four horizontal transfer genes and three antibiotic resistance genes (qacE disinfectants, aadA21 aminoglycoside, and drfA1 trimethoprim resistance genes) were present in another genomic island regions from 72848-80820. Other conjugative multi-resistance plasmids' genetic maps are shown in Figures $\mathrm{S} 8$ and $\mathrm{S} 9$.

\section{Effects of $\mathrm{PM}_{2.5}$ and $\mathrm{PM}_{10}$ on conjugative transfer}


Physiochemical characterization of $\mathrm{PM}_{2.5}$ and $\mathrm{PM}_{10}$ samples were described in Table S2, in the supporting information. $\mathrm{PM}_{2.5}$ and $\mathrm{PM}_{10}$ size distributions were 832 to $1096 \mathrm{~nm}$ and 2884 to $11482 \mathrm{~nm}$, respectively. Significant $(P<0.05)$ increases in transfer frequency fold changes occurred in the presence of $\mathrm{PM}_{2.5}$ and $\mathrm{PM}_{10}$ Fig. 5). For example, at $125 \mu \mathrm{g} / \mathrm{mL}, \mathrm{PM}_{2.5}$ and $\mathrm{PM}_{10}$ increased in the conjugative transfer rate by 1.3- and 2.1-fold, respectively, compared with that of control. Effects of $\mathrm{PM}_{2.5}$ and $\mathrm{PM}_{10}$ on ROS generation and cell membrane permeability were analyzed. ROS is considered to enhance the frequency of bacterial conjugative transfer [12]. Results of the present study showed that $\mathrm{PM}_{2.5}$ and $\mathrm{PM}_{10}$ induced a significant $(P<0.05)$ increase in donor intracellular ROS (up to 3.6- and 4.6-fold increases for $\mathrm{PM}_{2.5}$ and $\mathrm{PM}_{10}$, respectively, compared with that of control). TEM graphs showed the structure of bacteria during conjugation (Fig. S10).

\section{Genes expression during $\mathrm{PM}_{10}$ and $\mathrm{PM}_{2.5}$ exposure}

Exposure to all levels of $\mathrm{PM}_{10}$ and $\mathrm{PM}_{2.5}$ evidenced increased expression of conjugative processes relevant genes (Fig. 6). In this concern, ROS (ytfE, uspF, and napF), conjugation (ihfA and ompA) and pilus generations (htrE, sfmC, ybgP, yehB, and yra J) genes increased expression during PM exposure. Increased changes in SOS (yafO, recQ, umuC, sulA, and yebG) gene expression were also detected. Genes relevant to drug transmembrane transport ( $\mathrm{mdtM}$ and ompF) were upregulated. $\mathrm{PM}_{10}$ and $\mathrm{PM}_{2.5}$ further increased antibiotic response genes expression.

\section{Discussion}

This research provides a new perspective between air quality and human health. By integrating four-year data on resistance rates of ten clinic antibiotic-resistant pathogens and air quality, we compared the relationship between air quality and clinical antibiotic resistance across China. Significant relationship ( $P$ $<0.05)$ between air quality and antibiotic resistance was further demonstrated. We generated robust correlation tests based on four-year data sets, which are universal in most antibiotic-resistant bacteria. The robust correlation can be a powerful driver to an in-depth study of ARGs behavior in air environments.

ARGs in the clinical air environment were comprehensively characterized. ARGs from air may be mobilized and transferred to human commensals and abundant ARGs in $\mathrm{PM}_{10}$ and $\mathrm{PM}_{2.5}$ may infiltrate the respiratory system, causing an increased health risk to humans. In addition, air environments may be suitable ARGs carriers for long-distance propagation. A probable reason to explain the high abundance of ARGs and MGEs in air samples is that air environments contain low nutrition, high UV exposures, and multiple environmental pollution particles, which agree with previous reports demonstrating that such factors may increase abundance and transfer of ARGs in microbes $[29,39,40]$. In microbial taxa, Proteobacteria were mostly found in dust and air samples, whereas Firmicutes prevail in airway sputum samples, as reported by others [25]. Furthermore, daylight exposure can affect dust communities [41], particularly, Actinobacteria are associated with darkness and light exposure, which may be the reason for a higher abundance of Actinobacteria in ID samples than in other environments. Human beings are surrounded by dust and air, whose ARGs and bacteria content are relevant to public health. Moreover, the 
significant correlation between bacteria community and ARGs indicated bacterial communities may shape ARGs distribution and abundance in air.

Network analysis indicated co-spread of ARGs, MGEs, and bacteria among air, dust, and human sputum. Co-occurrence between fluoroquinolone resistance gene and Campylobacter spp., methicillin/ vancomycin resistance genes, and Staphylococcus spp. were observed. Campylobacter spp. and Staphylococcus spp. are zoonotic disease pathogens, exhibiting high levels of acquired antibiotic resistance, which has a worldwide public health impact [42-44]. In particular, fluoroquinolone-resistant Campylobacter spp. and methicillin/vancomycin-resistant Staphylococcus aureus are "high priority pathogens", according to the WHO; Campylobacter spp. causes serious diarrhea and Staphylococcus aureus is associated with skin infections, pneumonia, and bloodstream infections [45]. Methicillinresistant Staphylococcus aureus has previously been found in hospital and poultry farming air environments [46, 47]. Prevotella which is known to be resistant to tetracycline [48], shows strong associations with tetracycline resistance genes such as tetA-01, tet(36)-01, and tetPB-02; Veillonella, which is resistant to erythromycin and gentamicin, correlated to MLSB resistance genes (ermK-02) in the present study. Furthermore, Prevotella and Veillonella are commonly detected in airway sputum in high abundance; these genera can potentially influence airway microbiota composition by their metabolic products or by forming intergeneric coaggregates with other microbes[49-51]. Moreover, co-occurrence between these genera and MGEs (IS613, int12) were observed; MGEs may contribute to ARGs spread in the microbial community via horizontal gene transfer (HGT). In this regard, Campylobacter spp., Prevotella, multiple ARGs, and MGEs co-occurred in module I. This module may represent a typical community revealing the potential co-spread pattern of bacteria and ARGs in the environments, in which common bacteria may maintain the structure of community with pathogens and MGEs are more actively transferring resistance genes among microbial communities to resist environmental exposures.

Based on SourceTracker analysis, the relative contributions of bacteria and ARGs from $\mathrm{PM}_{2.5}$ samples are higher than that of other environmental samples, probably due to the increased infiltration of small particles to the airways. The relative contributions of environmental microbial to human airway sputum are higher than those of ARGs. It is reasonable that bacteria can be inhaled into human airways, whereas competitions from growth and reproduction may lead to the loss of antibiotic-resistant bacteria or loss of ARGs, and some extracellular ARGs degradation in human airways. Researchers have also noted that antibiotic resistance results in reduced fitness, compared with that of antibiotic-sensitive bacteria, which is known as the fitness cost $[52,53]$. Furthermore, most bacterial community and ARGs contributions in airway sputum are predicted to be of unknown origin, which may result from the large variety of individuals and other factor contributions, such as age, foodborne origin, home environment, personal care products, and different ecological conditions [54].

Importantly, conjugative multi-resistance plasmids were identified from the air. It seems possible that the plasmid can be transported by air, and transfer ARGs into human commensals at suitable conditions. pTAir-3 carried gene resistance to aminoglycoside, bleomycin, quinolone, tetracycline, trimethoprim, copper, and mercury. Plasmids carrying antibiotic and metal resistance genes allow bacteria to survive 
under difficult air conditions. Furthermore, pTAir-3 carried int11, transposons, type IV secretion systems and fimbrial genes, which related to conjugation transfer processes. This research provides a feasible and effective way to identify the conjugative multi-resistance plasmids in the air.

$\mathrm{PM}_{2.5}$ and $\mathrm{PM}_{10}$ were observed to enhance conjugation transfer efficiencies indicating that air pollutants promoted ARGs transfer between bacteria. Conjugation experiments were conducted in liquid solution which is a helpful method to identify the effect and mechanism of $\mathrm{PM}_{2.5}$ and $\mathrm{PM}_{10}$ on the horizontal transfer of ARGs [30]. These results are similar to those reported by Xie et al., 2019 [30]. The average daily inhaled air volume of for an adult is $20 \mathrm{~m}^{3}$, whereas the average lung pleural fluid volume is $20 \mathrm{~mL}$; when $\mathrm{PM}_{2.5}$ and $\mathrm{PM}_{10}$ concentration is $125 \mu \mathrm{g} / \mathrm{m}^{3}$ in air, the concentration of $\mathrm{PM}_{2.5}$ and $\mathrm{PM}_{10}$ in the lung fluid after 24-hour exposure will be $125 \mu \mathrm{g} / \mathrm{mL}$. In this scenario, conjugation between bacteria will be significantly enhanced. The results concerned us on the biological impact of air pollutants, particularly, ARGs spread.

We also investigated potential mechanisms to explain the role of $\mathrm{PM}_{2.5}$ and $\mathrm{PM}_{10}$ in the enhancement of conjugative transfer of RP4 plasmid. Multiple investigative approaches were used to reveal the underlying mechanisms, including flow cytometry and TEM to measure ROS response level and membrane permeability, and whole-genome RNA sequencing to evaluate gene expression. We verified the increased ROS level and membrane permeability with exposure to PM. ROS was activated in the presence of $\mathrm{PM}_{10}$ and $\mathrm{PM}_{2.5}$; increased ROS levels induces SOS, which damage cell membranes. This can weaken the barriers of cell membranes and promote horizontal ARGs dissemination [38]. RNA genomics revealed comprehensive gene expression of bacteria during PM exposure, which provides solid data to uncover the physiological changes of bacteria. Genes of pilus generation were upregulated on exposure to PM, which could facilitate physical cell-to-cell contact between donor and recipients, and enhance plasmid transfer. In addition, $\mathrm{PM}$ could increase the expression of genes response to antibiotic and drug transmembrane transport, augmenting bacteria antibiotic tolerance. Thus, $\mathrm{PM}_{2.5}$ and $\mathrm{PM}_{10}$ were confirmed to promote ARGs transfer.

\section{Conclusion}

In conclusion, this study demonstrates a significant relationship between resistance rates of ten clinic antibiotic-resistant pathogens and air quality. Abundant ARGs were found in clinical air, which may accelerate ARGs transfer between distant places. Importantly, conjugative multi-resistance plasmids and other MGEs in the air may frequently transfer between environments and within bacteria, exacerbating ARGs dissemination. In addition, $\mathrm{PM}_{2.5}$ and $\mathrm{PM}_{10}$ air pollutants were shown to increase $\mathrm{HGT}$ of ARGs. This research is major contribution to public health, involving the role of air pollutants in antibiotic resistance dissemination. Further studies could be applied to quantify the linkage between air pollutions and antibiotic resistance burden and analyze more air pollutant effects on HGT.

\section{Abbreviations}


ARGs

antibiotic resistance genes; MGEs:mobile genetic elements; PM:particulate matter; ARB:antibioticresistant bacteria; CREC:carbapenem resistant Escherichia coli; CRPA:carbapenem resistant Pseudomonas aeruginosa; CRAB:carbapenem resistant Acinetobacter baumannii; CRKP:carbapenem resistant Klebsiella pneumoniae; ERSP:erythromycin resistant Streptococcus pneumoniae; MRSA:methicillin resistant Staphylococcus aureus; MRCNS:methicillin resistant coagulase negative staphylococci; PRSP:penicillin resistant Streptococcus pneumoniae; QREC:quinolones resistant E. coli; 3GCREC:third-generation cephalosporin resistant E. coli; 3GCRKP:third-generation cephalosporin resistant Klebsiella pneumoniae; VREFS:vancomycin resistant Enterococcus faecalis; VREFM:vancomycin resistant Enterococcus faecium; AQI:air quality index; P:patient airway sputum samples; H:healthy human airway sputum samples; ID:indoor dust; OD:outdoor dust; I-PM2.5:indoor PM2.5; 0-PM2.5:outdoor PM2.5; I-PM10:indoor PM10; O-PM10:outdoor PM10; HT-qPCR:high-throughput qPCR; FCA:fluoroquinolone/ quinolone/ florfenicol/ chloramphenicol/ amphenicol; MLSB:macrolide/ lincosamide/ streptogramin B; QIIME:Quantitative Insights into Microbial Ecology; OTUs:operational taxonomic units; DGGE:denaturing gradient gel electrophoresis; TEM:Transmission electron microscopy; FPKM:fragments per kilobase of a gene per million mapped reads; LFC:log2 fold-change.

\section{Declarations}

\section{Ethics approval and consent to participate}

Sputum samples were obtained from volunteers in the Inpatient Respiratory Department with approval of the Zhejiang University School of Medicine ethics committee, and all subjects were provided with written informed consent before participation.

\section{Consent for publication}

Not applicable.

\section{Availability of data and materials}

The datasets used and analysed during the current study are available from the corresponding author on reasonable request.

\section{Competing interests}

The authors declare that they have no conflict of interest. 


\section{Funding}

This work was supported by Natural Science Foundation of China (21876147 and 21677121).

\section{Authors' contributions}

ZCZ and HC designed the study. ZCZ, YL, ZJL, LZ, LX, XYS, LXM and YJS performed experiments. ZCZ performed the bioinformatics and statistical analyses, and wrote the manuscript. $\mathrm{HC}$ reviewed the final manuscript. All authors read and approved the final manuscript.

\section{Acknowledgements}

We thank Prof. Junwen Li and Prof. Zhigang Qiu for providing E. coli with RP4 plasmid. We thank Bioultrastructure analysis Lab. of Analysis center of Agrobiology and environmental sciences, Zhejiang University for providing technical support on transmission electron microscopy.

\section{References}

1. WHO. Ten threats to global health in 2019. https://www.who.int/emergencies/ten-threats-to-globalhealth-in-2019.

2. Baquero F, Martinez JL, Rafael. Antibiotics and antibiotic resistance in water environments. Curr Opin Biotechnol. 2008; 19: 260-265.

3. Crane $M$, Watts $C$, Boucard T. Chronic aquatic environmental risks from exposure to human pharmaceuticals. Sci Total Environ. 2006; 367: 23-41.

4. D'Costa VM, King CE, Kalan L, Morar M, Sung WW, Schwarz C et al. Antibiotic resistance is ancient. Nature. 2011; 477: 457.

5. Zhou ZC, Zheng J, Wei YY, Chen T, Dahlgren RA, Shang X et al. Antibiotic resistance genes in an urban river as impacted by bacterial community and physicochemical parameters. Environ Sci Pollut R. 2017; 24: 1-10.

6. Zhu YG, Zhao Y, Li B, Huang CL, Zhang SY, Yu S et al. Continental-scale pollution of estuaries with antibiotic resistance genes. Nat Microbiol. 2017; 2: 16270.

7. Hirsch R, Ternes T, Haberer K, Kratz KL. Occurrence of antibiotics in the aquatic environment. Sci Total Environ. 1999; 225: 109-118. 
8. Guo J, Li J, Chen H, Bond PL, Yuan Z. Metagenomic analysis reveals wastewater treatment plants as hotspots of antibiotic resistance genes and mobile genetic elements. Water Res. 2017; 123: 468-478.

9. Gatica J, Tripathi V, Green SJ, Manaia CM, Berendonk T, Cacace D et al. High throughput analysis of integron gene cassettes in wastewater environments. Environ Sci Technol. 2016;11825.

10. Jia S, Shi P, Hu Q, Li B, Zhang T, Zhang XX. Bacterial Community Shift Drives Antibiotic Resistance Promotion during Drinking Water Chlorination. Environ Sci Technol. 2015; 49: 12271-12279.

11. Xu L, Ouyang W, Qian Y, Su C, Su J, Chen H. High-throughput profiling of antibiotic resistance genes in drinking water treatment plants and distribution systems. Environ Pollut. 2016; 213: 119-126.

12. Wang Y, Lu J, Mao L, Li J, Guo J. Antiepileptic drug carbamazepine promotes horizontal transfer of plasmid-borne multi-antibiotic resistance genes within and across bacterial genera. ISME J. 2019; 13: $509-522$.

13. Stevenson C, Hall JPJ, Harrison E, Wood A, Brockhurst MA. Gene mobility promotes the spread of resistance in bacterial populations. ISME J. 2017; 11: 1930-1932.

14. Pehrsson EC, Tsukayama P, Patel S, Mejíabautista M, Sosasoto G, Navarrete KM et al. Interconnected microbiomes and resistomes in low-income human habitats. Nature. 2016; 533: 212-216.

15. Zhu YG, Johnson TA, Su JQ, Qiao M, Guo GX, Stedtfeld RD et al. Diverse and abundant antibiotic resistance genes in Chinese swine farms. Proc Natl Acad Sci USA. 2013; 110: 3435-3440.

16. li GWS, Norton SB, Barnthouse LW. The Evolution of Frameworks for Ecological Risk Assessment from the Red Book Ancestor. Hum Ecol Risk Assess. 2003; 9: 1349-1360.

17. Xu WH, Zhang G, Zou SC, Li XD, Liu YC. Determination of selected antibiotics in the Victoria Harbour and the Pearl River, South China using high-performance liquid chromatography-electrospray ionization tandem mass spectrometry. Environ Pollut. 2007; 145: 672-679.

18. Gillings MR, Gaze WH, Pruden A, Smalla K, Tiedje JM, Zhu YG. Using the class 1 integron-integrase gene as a proxy for anthropogenic pollution. ISME J. 2014; 9: 1269-1279.

19. Gillings MR. Integrons: past, present, and future. Microbiol Mol Biol R. 2014;78: 257-277.

20. Pal C, Bengtsson-Palme J, Kristiansson E, Larsson DGJ. The structure and diversity of human, animal and environmental resistomes. Microbiome. 2016; 4: 54.

21. Yan C, Yang Y, Zhou J, Liu M, Nie M, Shi H et al. Antibiotics in the surface water of the Yangtze Estuary: Occurrence, distribution and risk assessment. Environ Pollut. 2003; 175: 22-29.

22. Jiang $L, H u X$, Yin $D$, Zhang $H, Y u Z$. Occurrence, distribution and seasonal variation of antibiotics in the Huangpu River, Shanghai, China. Chemosphere. 2011; 82: 822-828.

23. Li J, Zhou L, Zhang X, Xu C, Dong L, Yao M. Bioaerosol emissions and detection of airborne antibiotic resistance genes from a wastewater treatment plant. Atmos Environ. 2015; 124: 404-412.

24. Sung-Chul K, Kenneth C. Temporal and spatial trends in the occurrence of human and veterinary antibiotics in aqueous and river sediment matrices. Environ Sci Technol. 2007; 41: 50-57.

25. Zhou H, Wang X, Li Z, Kuang Y, Mao D, Luo Y. Occurrence and Distribution of Urban Dust-Associated Bacterial Antibiotic Resistance in Northern China. Environ Sci Tech Let. 2018; 5: 50-55. 
26. Zhou ZC, Feng WQ, Han Y, Zheng J, Chen T, Wei YY et al. Prevalence and transmission of antibiotic resistance and microbiota between humans and water environments. Environ Int. 2018; 121: 11551161.

27. Chen Q, An X, Li H, Su J, Ma Y, Zhu Y-G. Long-term field application of sewage sludge increases the abundance of antibiotic resistance genes in soil. Environ Int. 2016; 92-93: 1-10.

28. Turner S, Pryer KM, Miao VP, Palmer JD. Investigating deep phylogenetic relationships among Cyanobacteria and plastids by small subunit rRNA sequence analysis. J Eukaryot Microbiol. 1999; 46: 327-338.

29. Jiao YN, Chen H, Gao RX, Zhu YG, Rensing C. Organic compounds stimulate horizontal transfer of antibiotic resistance genes in mixed wastewater treatment systems. Chemosphere. 2017; 184: 53-61.

30. Xie S, Gu AZ, Cen T, Li D, Chen J. The effect and mechanism of urban fine particulate matter (PM2.5) on horizontal transfer of plasmid-mediated antimicrobial resistance genes. Sci Total Environ. 2019; 683: 116-123.

31. Bastian M, Heymann S, Jacomy M. Gephi: an open source software for exploring and manipulating networks. Proc Third Int ICWSM Conf (ICWSM). 2009; 8: 361-362.

32. Junker BH, Schreiber F. Correlation Networks. In Analysis of Biological Networks. Wiley-Interscience. 2008.

33. Knights D, Kuczynski J, Charlson ES, Zaneveld J, Mozer MC, Collman RG et al. Bayesian communitywide culture-independent microbial source tracking. Nat Methods. 2011; 8: 761.

34. Newman MEJ. The structure and function of complex networks. SIAM Rev. 2003; 45: 167-256.

35. Newman MEJ. Modularity and community structure in networks. Proc Natl Acad Sci USA. 2006; 103: 8577-8582.

36. Liu G, Zhang Y, van der Mark E, Magic-Knezev A, Pinto A, van den Bogert B et al. Assessing the origin of bacteria in tap water and distribution system in an unchlorinated drinking water system by SourceTracker using microbial community fingerprints. Water Res. 2018;138: 86-96.

37. Baral D, Dvorak BI, Admiraal DM, Jia S, Zhang C, Li X. Tracking the Sources of Antibiotic Resistance Genes in an Urban Stream during Wet Weather using Shotgun Metagenomic Analyses. Environ Sci Technol. 2018; 52: 9033-9044.

38. Yang K, Li L, Wang Y, Xue S, Han Y, Liu J. Airborne bacteria in a wastewater treatment plant: Emission characterization, source analysis and health risk assessment. Water Res. 2019; 149: 596-606.

39. Di CA, Fontaneto D, Doppelbauer J, Corno G. Fitness and recovery of bacterial communities and antibiotic resistance genes in urban wastewaters exposed to classical disinfection treatments. Environ Sci Technol. 2016; 50: 10153-10161.

40. Qiu Z, Yu Y, Chen Z, Jin M, Yang D, Zhao Z et al. Nanoalumina promotes the horizontal transfer of multiresistance genes mediated by plasmids across genera. Proc Natl Acad Sci USA. 2012; 109: 4944-4949. 
41. Fahimipour AK, Hartmann EM, Siemens A, Kline J, Wymelenberg KVD. Daylight exposure modulates bacterial communities associated with household dust. Microbiome. 2018; 6: 175.

42. Van Boeckel TP, Glennon EE, Chen D, Gilbert M, Robinson TP, Grenfell BT et al. Reducing antimicrobial use in food animals. Science. 2017; 357: 1350-1352.

43. Dunphy R. Quantitative Campylobacter spp., antibiotic resistance genes, and veterinary antibiotics in surface and ground water following manure application: Influence of tile drainage control. Sci Total Environ. 2015; 532: 138-153.

44. Berendonk TU, Manaia CM, Christophe M, Despo FK, Eddie C, Fiona W et al. Tackling antibiotic resistance: the environmental framework. Nat Rev Microbiol. 2015; 13: 310-317.

45. Willyard C. The drug-resistant bacteria that pose the greatest health threats. Nature. 2017; 543: 15.

46. Liu D, Chai T, Xia X, Gao Y, Cai Y, Xiaoxia LI et al. Formation and transmission of Staphylococcus aureus (including MRSA) aerosols carrying antibiotic-resistant genes in a poultry farming environment. Sci Total Environ. 2012; 426: 139-145.

47. Saadoun I, Jaradat ZW, Tayyar IAA, Nasser ZE, Ababneh Q. Airborne methicillin-resistant Staphylococcus aureus in the indoor environment of King Abdullah University Hospital, Jordan. Indoor Built Environ. 2014; 24: 315-323.

48. Arzese AR, Tomasetig L, ., Botta GA. Detection of tetQ and ermF antibiotic resistance genes in Prevotella and Porphyromonas isolates from clinical specimens and resident microbiota of humans. J Antimicrob Chemoth. 2000; 45: 577-582.

49. Salzman NH, Hung K, Haribhai D, Chu H, Karlsson-Sjöberg J, Amir E et al. Enteric defensins are essential regulators of intestinal microbial ecology. Nat Immunol. 2009; 11: 76-83.

50. Gronow S, Welnitz S, Lapidus A, Nolan M, Ivanova N, Glavina Del Rio T et al. Complete genome sequence of Veillonella parvula type strain (Te3T). Stand Genomic Sci. 2010; 2: 57-65.

51. Man WH, Wa DSP, Bogaert D. The microbiota of the respiratory tract: gatekeeper to respiratory health. Nat Rev Microbiol. 2017; 15: 259-270.

52. Melnyk AH, Wong A, Kassen R. The fitness costs of antibiotic resistance mutations. Evol Appl. 2015; 8: 273-283.

53. Maharjan R, Ferenci T. The fitness costs and benefits of antibiotic resistance in drug-free microenvironments encountered in the human body. Env Microbiol Rep. 2017; 9: 635-641.

54. Zou S, Xu W, Zhang R, Tang J, Chen Y, Zhang G. Occurrence and distribution of antibiotics in coastal water of the Bohai Bay, China: Impacts of river discharge and aquaculture activities. Environ Pollut. 2011; 159: 2913-2920.

\section{Figures}



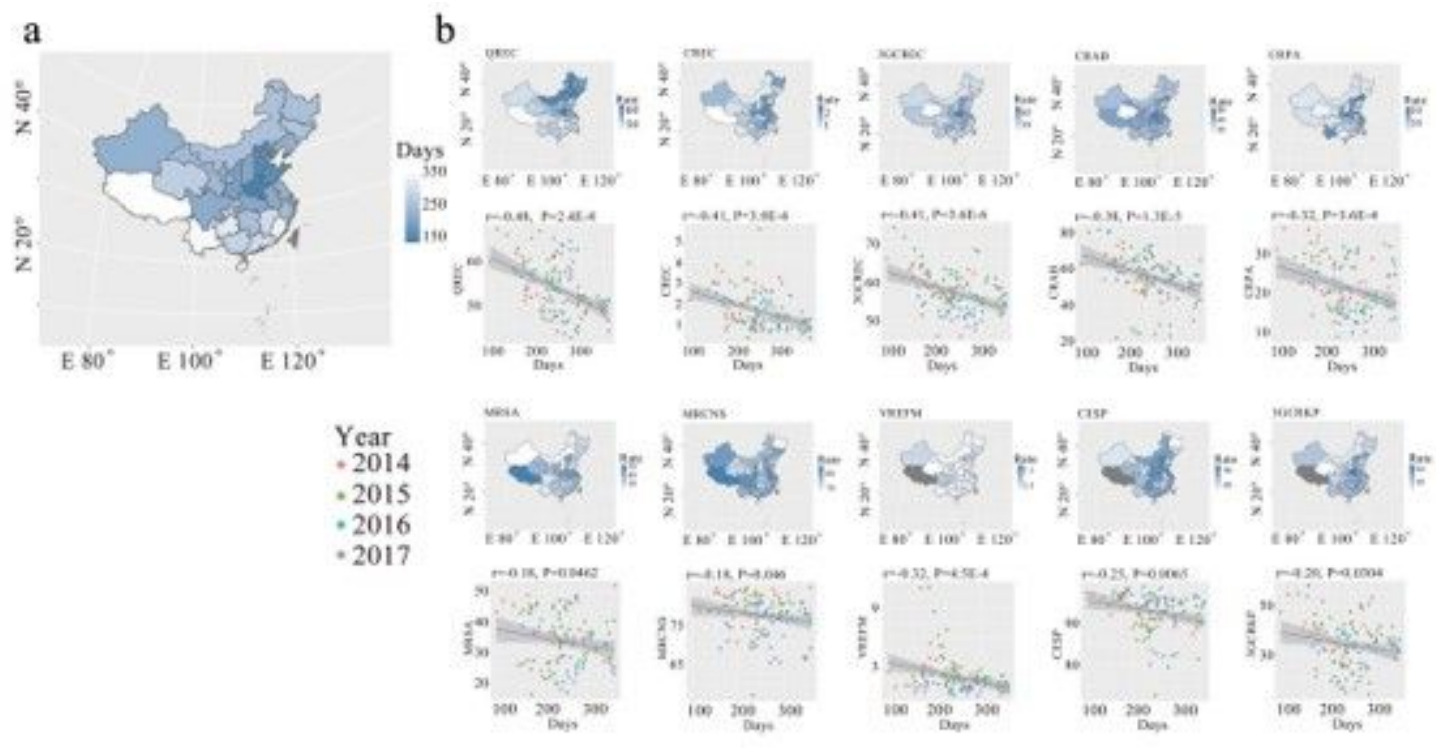

\section{Figure 1}

Linkages between antibiotic resistance and air quality. a, A heat map of the 4-year average number of days when air pollution index is below 100 across China. b, heat maps of the carbapenem resistant Escherichia coli (CREC), Pseudomonas aeruginosa (CRPA) and Acinetobacter baumannii (CRAB), erythromycin resistance Streptococcus pneumoniae (ERSP), methicillin resistant Staphylococcus aureus (MRSA), and coagulase negative staphylococci (MRCNS), quinolones resistance E. coli (QREC), thirdgeneration cephalosporin resistant E. coli (3GCREC) and Klebsiella pneumoniae (3GCRKP), and vancomycin resistance Enterococcus faecium (VREFM) across China and mean antibiotic resistance versus average number of days for the antibiotic resistance pathogens, unadjusted weighted linear relationships for the years 2014- 2017 are shown. Gray, no data reported.

a

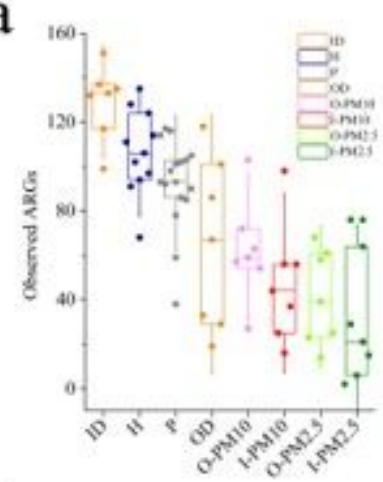

d

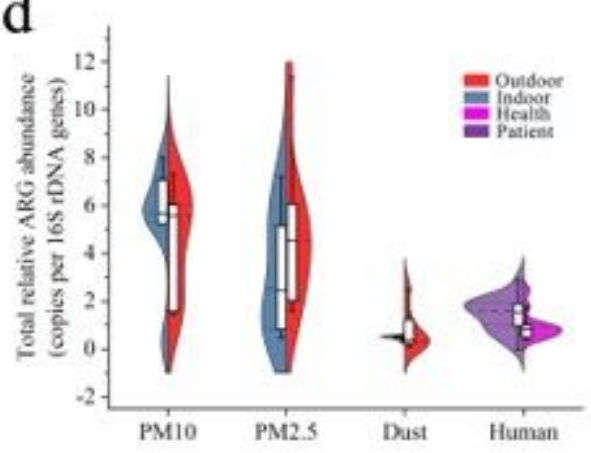

b

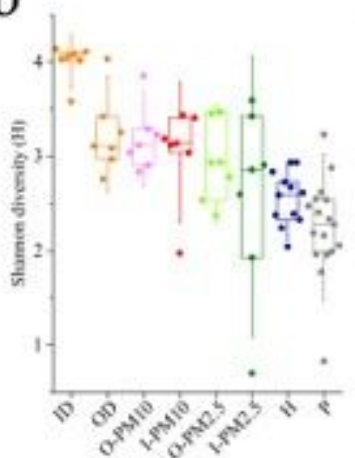

e c

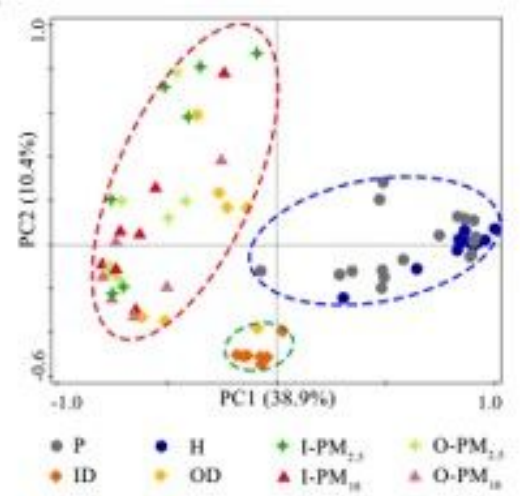

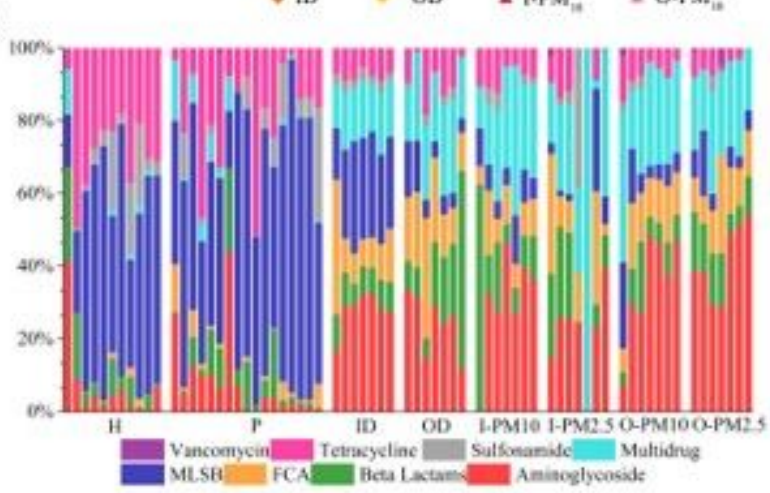

Page $17 / 20$ 


\section{Figure 2}

Profiles of antibiotic resistance genes. a, b, Number and diversity of ARGs in each sample (error bars mean s.d.; centre bars mean median). c, Principal coordinate analysis showing the distribution of ARGs profiles using the Bray-Curtis dissimilarity index. $d$, Violin plot showing the median and quartile ranges (box and whiskers) as well as the density distribution (shaded area) of the total relative abundance of all antibiotic resistance genes, red represents outdoor samples, cyan represents indoor samples, purple represents patient airway samples, pink represents health human airway samples. e, Bar graph showing the relative abundance of resistance genes to different classes of antibiotics across environments. Patient airway sputum $(P)$, healthy human airway sputum $(H)$, indoor dust (ID), outdoor dust (OD), indoor PM2.5 (I-PM2.5), outdoor PM2.5 (O-PM2.5), indoor PM10 (I-PM10) and outdoor PM10 (O-PM10).
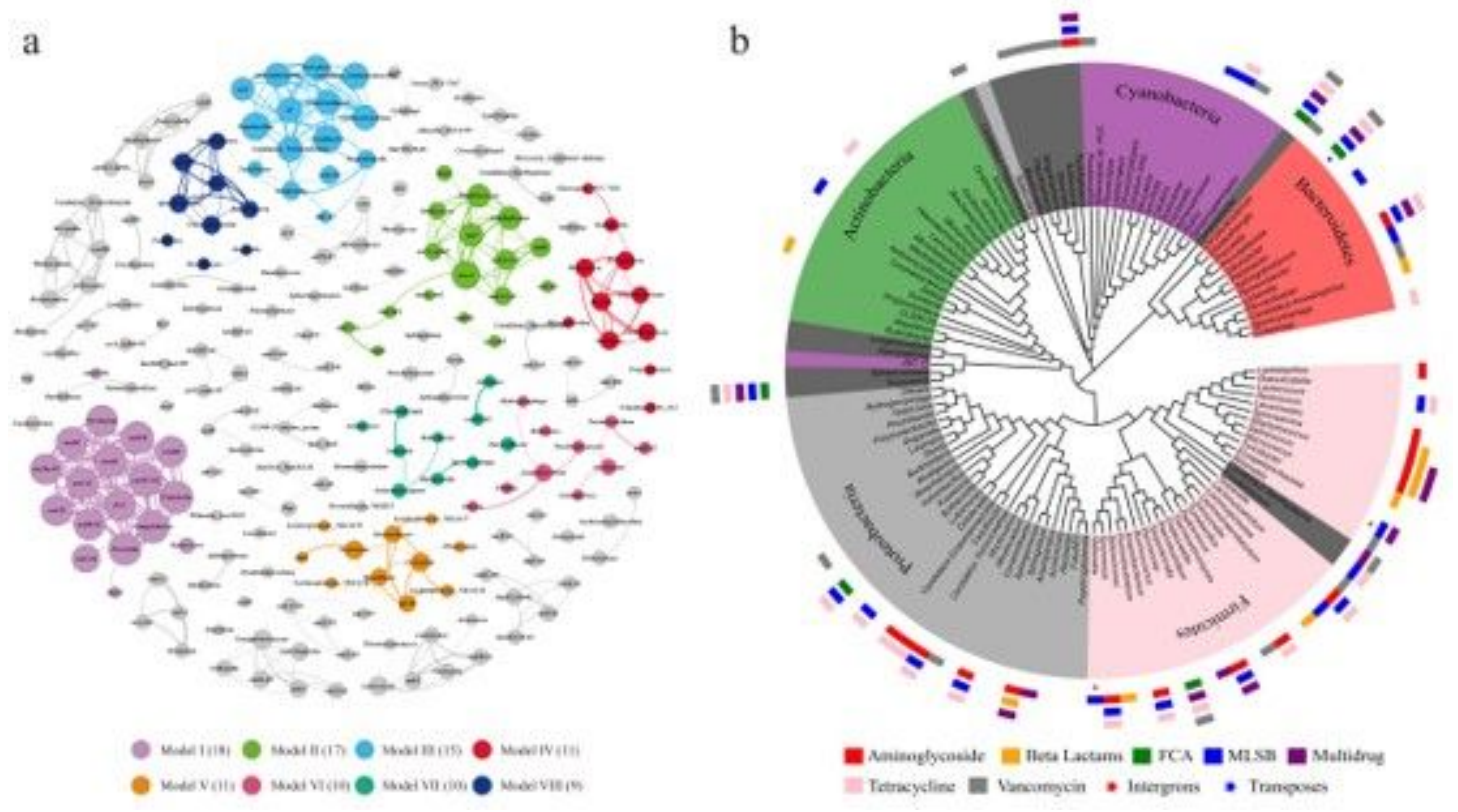

\section{Figure 3}

Co-occurrence patterns of ARGs, MGEs and bacteria. a Network analysis, the nodes were coloured according to modular classes. A connection represents a strong (Spearman's correlation coefficient $(\rho)>$ $0.9)$ and significant $(P<0.01)$ correlation. Node size was weighted according to the number of connections (that is the degree) and edges weighted according to the correlation coefficient. b, Phylogenetic tree of 124 taxa (that are nodes of bacteria from the Network) using a maximum likelihood method, with small colour stars and strips indicating the classes of MGEs and ARGs. 


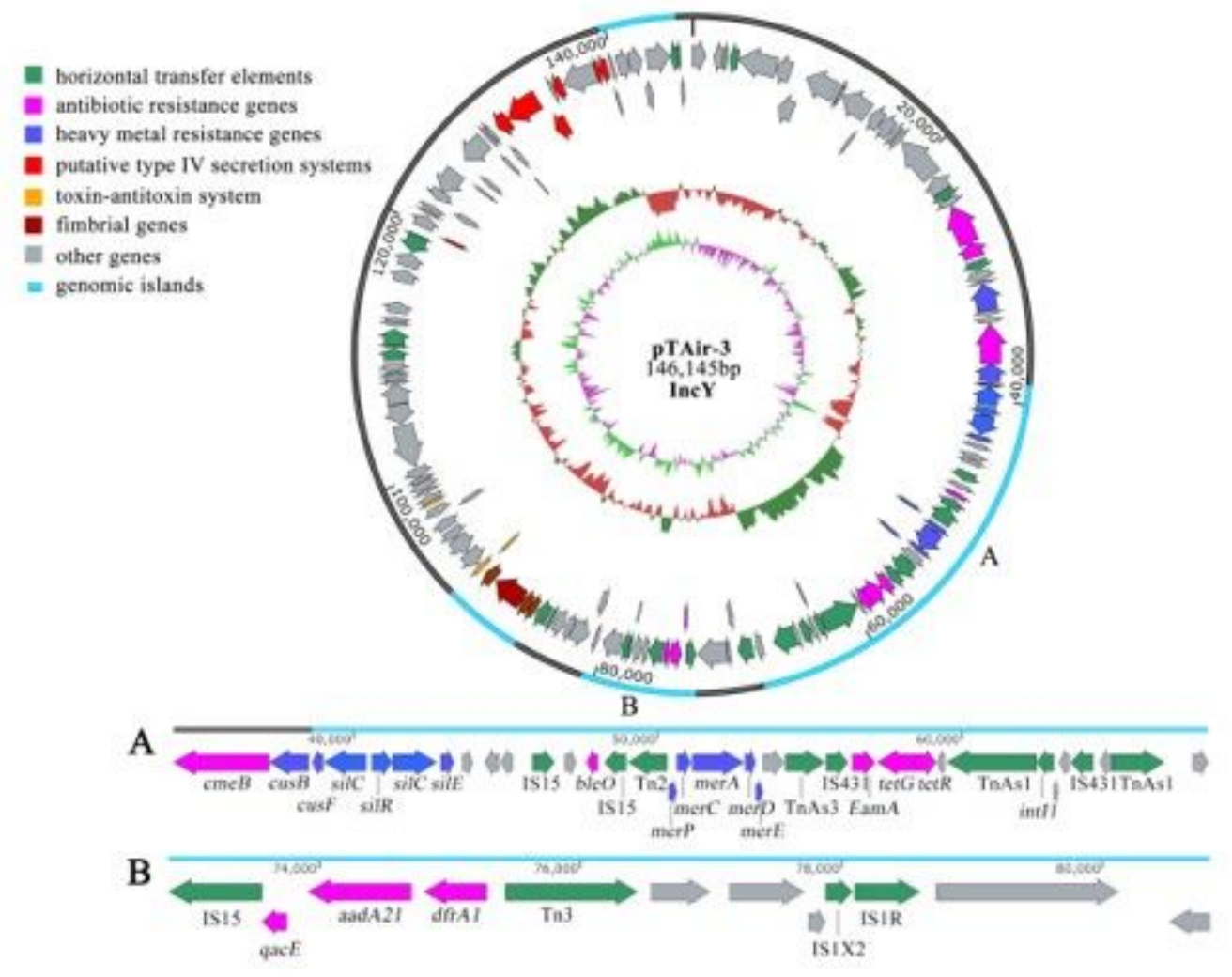

\section{Figure 4}

Genomic map of the conjugative multi-resistant plasmid pTAir-3 obtained from air. Positions of gene classes are indicated by colour. The inner two rings represent GC content and GC skew. a, b, Expanded view of the putative genomic island consisting of antibiotic and mental resistance gene and MGEs.

a

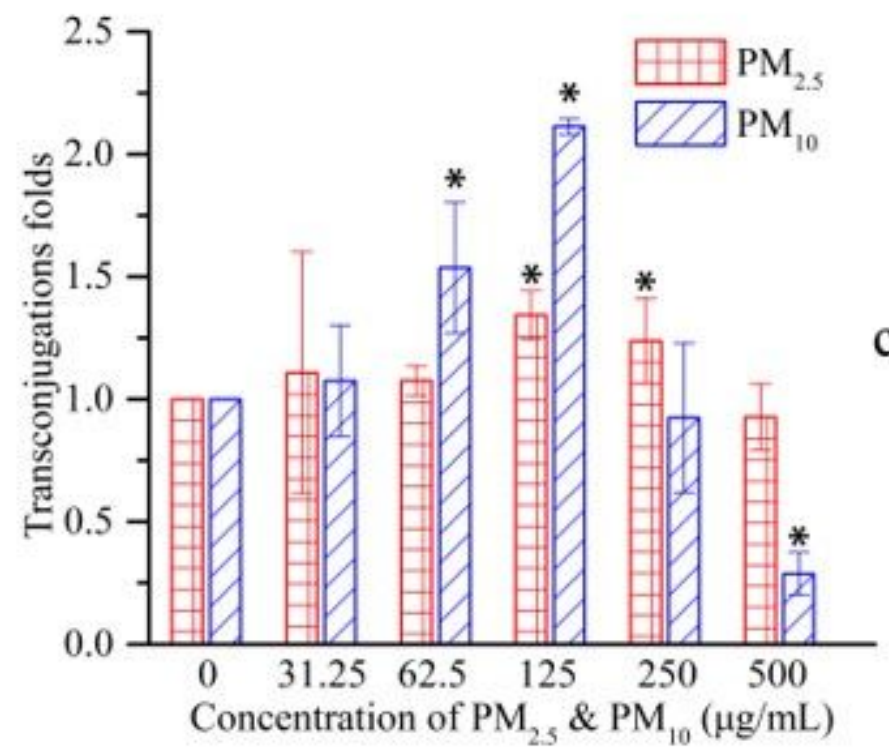

b
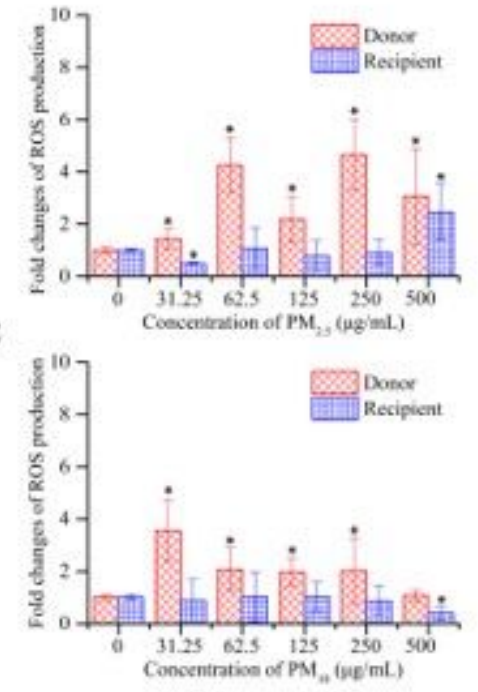

Figure 5 
Conjugation transfer efficiency and ROS response level. a, Fold changes of conjugative transfer frequency. b, Fold changes of ROS production by PM2.5. c, Fold changes of ROS production by PM10.

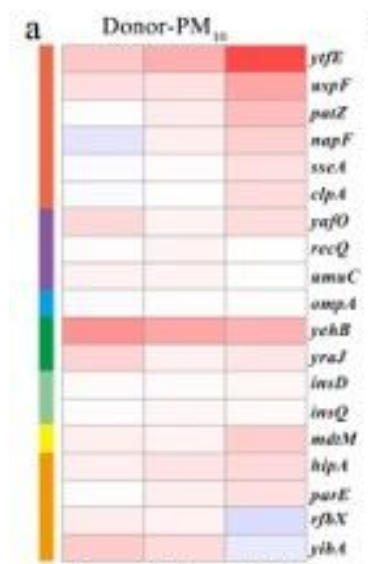

Low Medium High

I ROS Conjugation

I sos Pilus generation

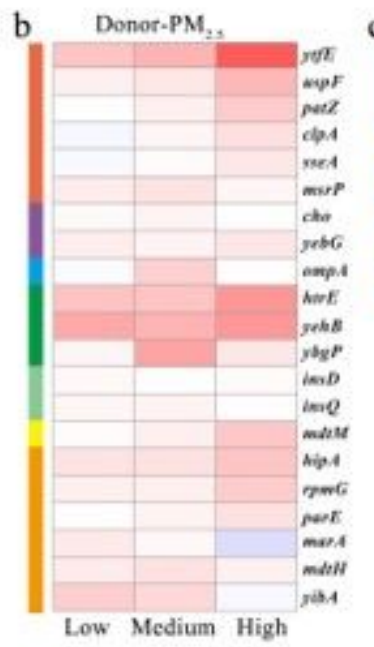

II Transposition
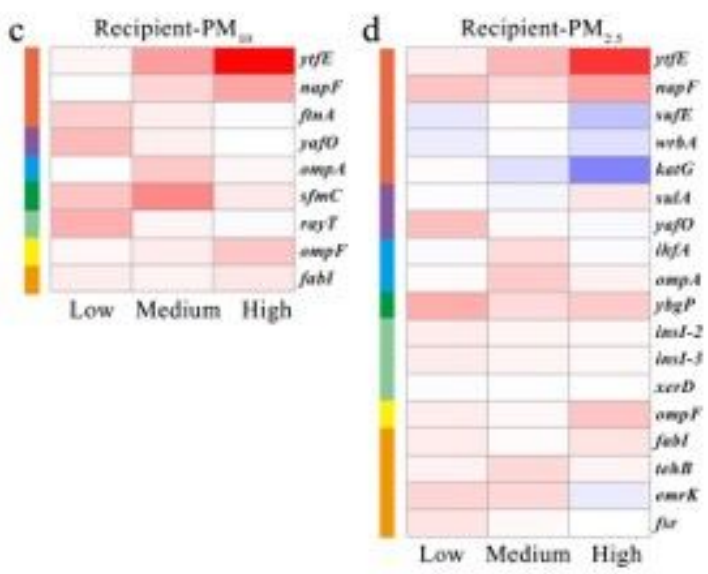

Figure 6

Gene expression level. a, Fold changes of expression of genes in donor bacteria with exposure to PM10. b, Fold changes of expression of genes in donor bacteria with exposure PM2.5. c, Fold changes of expression of genes in recipient bacteria with exposure to PM10. d, Fold changes of expression of genes in recipient bacteria with exposure to PM2.5. Low, medium, and high refer to $31.25,125.0$, and 500.0 $\mu \mathrm{g} / \mathrm{mL}$, respectively.

\section{Supplementary Files}

This is a list of supplementary files associated with this preprint. Click to download.

- Supportinginformation.docx 